\title{
Performance Evaluation of Best Path Selection using Multi Hop Decode and Forward Cooperative system
}

\author{
M.Bhaskara, Christeena Joseph
}

\begin{abstract}
Abstract: In this method, examined that Multibounce correspondence is the most ideal route for improving the inclusion region with decreased transmitted power. From this, we can without much of a stretch finding a way determination dependent on multi-jump translate and forward $(\mathrm{DF})$ agreeable framework. At that point, it is said to be a basic parallel multibounce ways based agreeable correspondence framework. Assumed all jumps on the whole agreeable ways are presenting to allowed Rayleigh blurring. Picking the better way in the greatest multi-jump interface between the source and goal is determined by choosing the max-min determination criterion. The transfers which are found in the chose helpful way to utilize the image astute DF convention in the demodulate to get the information from the previous hub with the assistance of image astute way and it is sent to the succeeding hub. A systematic structure is progressed to locate an exact blunder execution of the given framework. In ongoing we determine a right articulation for the normal piece blunder rate of the maximum min foundation based upon the best way choice plan, all together with the united power arrangement. The coding gain and the assorted variety request of the plan are moreover determined with the assistance of the explanatory strategy.
\end{abstract}

Keywords: Broad-cast, channel hopping, a cognitive radio network, co-operative communication, decode and forward protocol, Langford pairing, multihop transmitting, route range, performance calculation.

\section{INTRODUCTION}

Multi-bounce founded agreeable correspondence framework exists valuable for expanding the communication go (inclusion) of a remote correspondence framework. Disentangle and-forward (DF) convention requires advanced preparing at the transfers and maintains a strategic distance from mistake amassing in opposition to the enhance also a forward convention. Be that as it may, a multi-jump DF helpful framework is very inclined to mistake spread because of the nearness of different incorrect handing-off hubs. This issue restrains the augmentation of the DF convention to multi-bounce frameworks. The execution investigation of multi-bounce DF transferring has been anticipated. An upper destined for the blunder likelihood of the multi-bounce decode and forward framework is explored then the idea of multi-bounce covered variety is presented. The normal blunder execution of the multi-jump created decode and forward convention is broke down. However, all previously mentioned works over multi-jump handing-off

Revised Manuscript Received on September 10, 2019.

M.Bhaskara, Student, Department of ECE, Saveetha Institute of Medical and Technical Sciences, Chennai, Tamilnadu, India.

(Email: mbhaskar960@gmail.com).

Dr. Christeena Joseph, Associate professor, Department of ECE, Saveetha Institute of Medical and Technical Sciences, Chennai,Tamilnadu,India.

(Email: christeena003@gmail.com). think about that there is just a solitary multi-bounce way accessible between the source and goal. Since the DF convention loses decent variety on account of incorrect handing-off, the execution of DF convention in a multi-jump organize is seriously influenced. So as to improve the blunder and assorted variety execution of the DF convention based multi-bounce correspondence framework, different multi-jump ways can be conveyed among the user and goal. The transmission of the user information, a finest multijump way be able to be picked. The way determination created multi-jump framework becomes developed decent variety, be that as it may, the blunder examination of a way determination based multi-jump arrange is a troublesome issue. The blackout examination of the ideal way choice in multi-bounce organize containing a transfer in each jump is played out the blackout likelihood of a helpful multi-jump parallel hand-off system is performed. Diverse way choice strategies for a multi-bounce coordinate with various ways have been proposed and thought about by utilizing reproductions A rough lesser assured of the bit error rate of the maximum-minimum paradigm founded way determination remains gotten through the principal creator completed Rayleigh blurring networks Successively, a similar creator stretched out the investigation towards the Nakagami-m blurring networks Near the finest of insight, the accurate (otherwise near precise) mistake amount of a way determination created multi-jump decode and forward framework isn't altogether examined so far in the writing. To be increasingly explicit there is no realized endeavour to determine the accurate or near precise logical mistake amount of the system choice founded multi-jump conspire in the writing up until this point. In this method, think about the maximum-minimum determination basis generally advantageous way choice in a multihop DF helpful framework in Rayleigh blurring. We present a numerical structure for the examination of the normal BER of the way choice based multihop plot. The normal BER of the considered determination conspire is inferred under Rayleigh blurring as far as power arrangement. By utilizing the determined BER articulation, the coding increase and decent variety request of the way determination plot are systematically got. By utilizing numerical precedents, it is watched that the proposed BER articulation gives an exceptionally near precise portrayal of the considered multihop framework. 


\section{METHODOLOGY}

\section{System model:}

\section{Performance Analysis Of Finest Route}

In this method, a decode and forward agreeable framework with $\mathbf{J}$ similar helpful ways between the source and destination is considered, what's more, the goal, as appeared Every way contains $\mathrm{N}-1$ transfers with the end goal that there exist $\mathrm{N}$ jumps in an agreeable way. A solitary agreeable way is chosen for transmission of the information of the source to the goal. The transfers in the chose agreeable way to utilize the image insightful DF convention, in which they demodulate the got information after the previous hub in an image shrewd way furthermore, forward it without utilizing slightly onward blunder remedy coding towards the following hub. allow hi, j, $\gamma \mathrm{i}, \mathrm{j}$, and ${ }^{-} \gamma \mathrm{i}, \mathrm{j}$ indicate the Gaussian disseminated compound esteemed station coefficient through zero mean and $\sigma 2 \mathrm{I}, \mathrm{j}$ difference, the quick flag to-commotion proportion signal to noise ratio, and the normal signal to noise ratio, separately, of the $\mathrm{jth}, \mathrm{j}=$ $1, \ldots, \mathrm{N}$, a jump of the ith, $\mathrm{I}=1, \ldots, \mathrm{J}$, helpful way. At that point, we have the relations $\gamma \mathrm{i}, \mathrm{j}=|\mathrm{hi}, \mathrm{j}| 2 / \sigma 2$ plus ${ }^{-} \gamma \mathrm{i}, \mathrm{j}=$ $\sigma 2 \mathrm{i}, \mathrm{j} / \sigma 2$, where $\sigma 2$ speaks to the normal intensity of the complex-esteemed zero-mean added substance white Gaussian clamor. In the event that the maximum-minimum paradigm is utilized For best-way determination, at that point the way chose for helpful transmission contains the best jump, through the immediate signal to noise ratio signified by $\gamma$ max,min, which is picked as Consequently, the

$$
\left.\gamma_{\max , \min } \triangleq \max \left(\left.\min \left(\gamma_{i, j}\right)\right|_{j=1} ^{N}\right)\right|_{i=1} ^{J},
$$

In this way, the best way contains the jump with the most elevated estimation of the least of immediate jump SNRs in every way. The best way choice based multi-jump handingoff is relevant to several pragmatic situations, e.g., psychological radio systems also open-space visual systems. Moreover, for the finest-way determination, both the source before the goal need cover the data of the momentary signal to noise ratio of everything bounces. The likelihood thickness work of $\gamma \max$, min is communicated such as

$$
f_{\gamma_{\max , \text { min }}}(\gamma)=e^{-b \gamma} \sum_{k=0}^{\infty}(J+k-b \gamma) g_{k} \gamma^{J+k-1}, \quad \gamma \geq 0
$$

\section{Scheme in decode and forward protocol :}

Here, to investigate the normal bit error rate execution of the finest way determination conspire now the multi-jump decode and forward helpful framework utilizing parallel stage move keying (BPSK) star grouping.

Under the condition that the ith helpful way is chosen for collaboration, the normal BER of the way determination plot is given by underestimation of over the channel rises of all jumps in the ith agreeable way. Since troupe be around exists distributive, consider the minimization of in a timethrough-time way.

$$
\begin{aligned}
& P_{e_{\mathrm{i}}}^{c}\left(\gamma_{1}, \gamma_{2}, \ldots, \gamma_{N}\right) \\
& =\prod_{t=1}^{N}\left(1-P_{e_{i, j}}\right)
\end{aligned}
$$

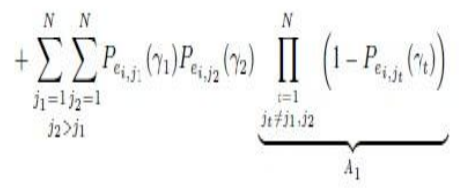

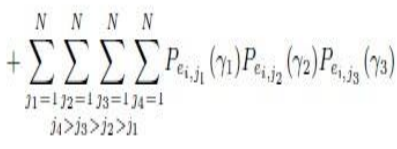

\section{Marginalization of $\mathrm{J} 1$ :}

Consequently, the $\mathrm{j} 1$ th bounce in the ith agreeable way can be the MMH or some other jump among the remaining $\mathrm{N}-1$ jumps in an ith way is the MMH. In light of this perception, the minimization of $\mathrm{J} 1$, under the condition that the ith agreeable way is chosen for collaboration, can be spoken to as where $\mathrm{Q}(\cdot)$ is the Gaussian Q-work. Give us now a chance to assess the terms $\mathrm{V}(1)(\mathrm{i}, \mathrm{j} 1)$ and $\mathrm{V}(2)(\mathrm{i}, \mathrm{j} 1)$ independently. The term V $(1)(\mathrm{i}, \mathrm{j} 1)$ can be communicated as integrals by

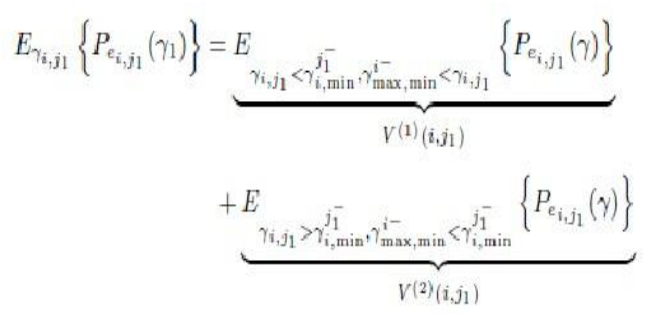

\section{Marginalization of JN}

For minimization of $\mathrm{JN}$, we see that mistakes in all $\mathrm{N}$ interfaces in an ith way are considered here; along these lines, there exist $\mathrm{N}$ conceivable commonly selective occasions where every last one of the $\mathrm{N}$ connects in the ith helpful way can be the MMH.

$$
\begin{aligned}
& V^{(1)}\left(i, j_{1}, j_{2}, \ldots, j_{N}\right) \\
& =\frac{1}{\bar{\gamma}_{i, j_{1}} \bar{\gamma}_{i, j_{2}}, \ldots, \bar{\gamma}_{i, j_{N}}} \int_{x_{1}=0}^{\infty} Q\left(\sqrt{2 x_{1}}\right) \\
& x e^{-\frac{x_{1}}{\gamma_{i}, j_{1}}} \int_{x_{2}=x_{1}}^{\infty} Q\left(\sqrt{2 x_{2}}\right) e^{-\frac{x_{2}}{\gamma_{i, j}}}, \ldots, \int_{x_{N}=x_{1}}^{\infty} Q\left(\sqrt{2 x_{N}}\right) \\
& \times e^{-\frac{x_{N}}{\gamma_{i, j N}}} \int_{w=0}^{x_{1}} f_{\gamma_{\max , \min }}(w) d x_{1} d x_{2}, \ldots, d x_{N} d w .
\end{aligned}
$$

\section{Coding Gain And Diversity Order Of The Best-Path :}

Selection Scheme: where $\mathrm{dk}=\mathrm{Nk}+1 /(\Gamma(\mathrm{k}+2))$, and the superscript $\mathrm{y}\{\mathrm{x}\} \mathrm{k}$ implies that $\mathrm{yk}$ is $\mathrm{x}-$ multiple times with itself, e.g., $\mathrm{y}\{2\} \mathrm{k}=\mathrm{yk} * \mathrm{yk}$. 
By watching the way that $2 \mathrm{~F} 1(\mathrm{a}, \mathrm{b} ; \mathrm{c} ; \mathrm{z}) \rightarrow 1$ for $\mathrm{z} \rightarrow 0$, it tends to appear from that at a high SNR, the normal BER of the best-way choice plan is approximated by the streamlined structure, i.e.,

$$
P_{e} \approx J N \sum_{k=0}^{\infty} \frac{d_{k}^{\{J-1\}} \Gamma(J+k+1 / 2)}{2(J+k) \sqrt{\pi}} \gamma^{-J-k} .
$$

It very well may exist realized after that the normal bit error rate articulation covers summation expressions, every relative towards ${ }^{-} \gamma-\mathrm{J}-\mathrm{k}$. Along these lines, by a very expensive signal to noise ratio, the time with the most reduced intensity of ${ }^{-} \gamma$, i.e., ${ }^{-} \gamma-\mathrm{J}$, drive command the rot of Pe; the coding count and decent variety request of the best way determination plan be able to be resolved after this period it was. Subsequently, the normal likelihood of mistake assumed now be able to stay approached at an exceptionally great and coding gain, separately, are

$$
G_{d}=J \text { and } G_{c}=\left(\frac{2 \sqrt{\pi}}{\Gamma(J+1 / 2) N^{j}}\right)^{\frac{1}{7}} \text {. }
$$

\section{EXISTING SYSTEM}

\section{Matlab coding:}

MATLAB is the best language used for the specified process. MATLAB incorporates design, encoding, as well as an illustration in information to understand condition wherever problems, as well as measures, stay connected in open scientific documentation. MATLAB consists of intellectual outline whose fundamental data factor stays a demonstration that organizes not involve dimensioning. This enables the user towards comprehend numerously specified process problems, mostly individuals through the framework as would well as direction activities, in fewer phase than this one yield towards constituting a program in a scalar nonintuitive language, for example, $\mathrm{C}$ or FORTRAN. MATLAB best part a collection of application explicit preparations which stay called tool kits. The aforementioned is critical towards greatest clients of MATLAB that device compartments authorization close learn and relate certain innovation. The device kits stay complete increases of MATLAB capabilities, purported records that stretch out the MATLAB condition towards settle specific classes of problems. MATLAB stays a grid-constructed encoding instrument. In spite of the information that structures commonly need not remain dimensioned expressly, the user consumes regularly near examine cautiously designed for framework measurements.

\section{EXAMPLE}

clc

closeall

clearall

$\% \%$ Systematic plus replicated mistake presentation of the finest route range

$\mathrm{SNR}=0: 5: 35$;

$\mathrm{j}=2$;

$\mathrm{N}=5$; channelcoffient $=0$;

$\mathrm{v}=\operatorname{var}(\mathrm{SNR}, 1)^{\wedge} 2 ; \%$ variance

$\%$ BPSK constellation

c1 =rayleighchan $(1 \mathrm{e}-12,120)$;

$\%$ c1.PathDelays $=[0$ le-6];

$\%$ Gaussian distributed complex-valued

$\mathrm{y}=\operatorname{awgn}(\mathrm{SNR}, \mathrm{N}$, 'measured');

$\% \mathrm{Q}$ function

End

Now the result that it isn't considered approximately different, the normal grid shows binary capacities $(n \times m$.) Fragment directions and support courses stay pronounced towards dependably through $(\mathrm{n} \times 1)$ and $(1 \times \mathrm{n})$ networks, individually. MATLAB activities characterized keen on the accompanying kinds of tasks number-crunching then legitimate tasks, numerical capacities, graphical and input/yield tasks.

\section{TEST AND RESULT}

On behalf of direct problems, incoming difficulties by the MATLAB brief in the direction space stands fast also, creative. Be that as it may, as the amount of directions increases, otherwise at whatsoever topic a difference in the esteem of at least one factors through a verification is wanted, composing on the MATLAB passing moves toward becoming repetitive. MATLAB permits to put MATLAB directions in a simple content document, as well as by advising MATLAB to undeveloped this record, the putaway directions stand assessed single-via-single equally still they stood simply composed in. Individuals proceedings exist named MATLAB documents.

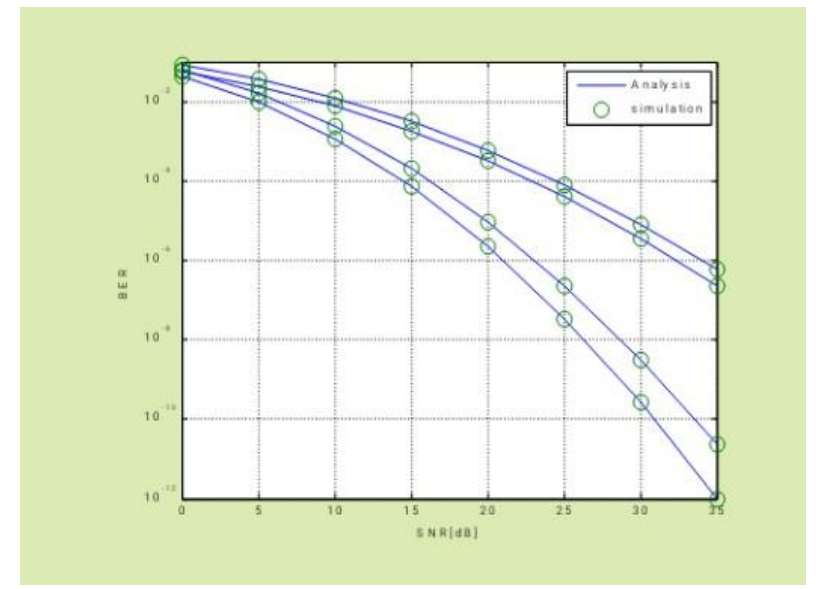

Fig 1; Systematic and replicated mistake presentation of the finest-route selection scheme using $\gamma \overrightarrow{\mathbf{l}}, \mathbf{j}=\bar{\gamma} \mathbf{s}, \mathbf{d}=\bar{\gamma}$.

Here exist binary kinds of MATLAB documents: Characters, which don't accept input arguments otherwise arrival produce arguments. Tasks, which recognize input arguments and arrival produce controversies. Interior factors remain a region to the capability.the key distinction in the sentence arrangement of a Characters-text also a Purposebest is the main route. 


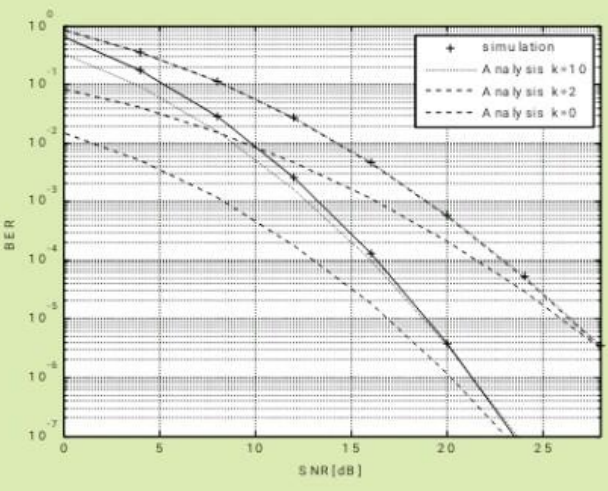

Fig 2: Replicated and systematic bit error rate versus signal to noise ratio presentation of the finest route range outline using $\gamma \overrightarrow{\mathbf{i}}, \mathbf{j}=\bar{\gamma} / \mathbf{j}$.

The primary track in a Purpose document starts through the catchphrase work pursued in the depressed of produce controversies, a parallels symbol, the designation of the capability as well as consummation through the depressed of info factors covered in brackets also insulated through commas. On the off chance that the capability has various produce controversies, the produce controversy grade need stand covered in four-sided sections, for example, grind $[\mathrm{x}, \mathrm{y}, \mathrm{z}]=$ cosytrans (theta, phi, rho) In a Script-record around stays not at all predefined language arrangement for the primary track.

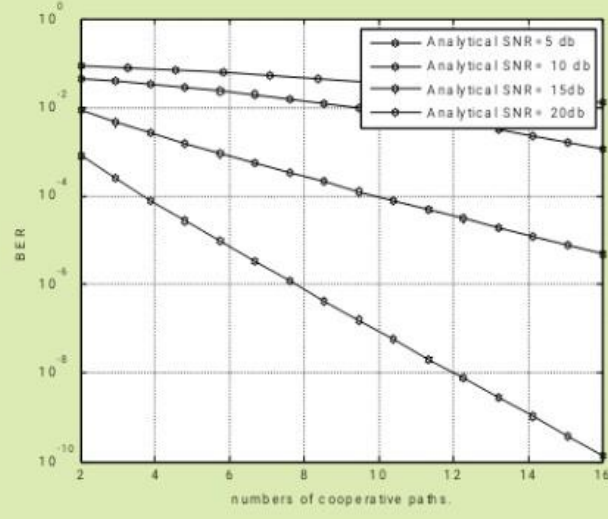

Fig 3: Systematic and replicated bit error rate of the finest route range outline for diverse quantities of supportive paths.

Towards mark before modifying a file in the world of Linux the direction it can stay applied to start a word processor. The direction necessity stays composed of data Linux order window. The use of this reporting superior remains basic also the greatest vital directions be able to exist originate in the best down-menus. Another imperative angle regarding the effort through MATLAB must remain referenced at this point. The directions displayed in this method stayed unfilled without a concluding semicolon.

\section{PROS AND CONS}

In this method, researched that the execution of multibounce audio frequency-DF FDR frameworks which improves the unearthly effectiveness contrasted and HDR.
Multi-jump steering is a kind of correspondence in radio systems in which organize inclusion zone is bigger than radio scope of single hubs. In this manner, to achieve the goal a hub can utilize different hubs as transfers. The handing-off framework assumes a noteworthy job in improving the correspondence. Summed up recurrence division multiplexing (GFDM) is another idea utilized in 5G cell framework which gives the preferred execution over OFDM. The plan depends on the sifted multicarrier approach which offers expanded adaptability. Consequently,, GFDM offers a higher information rate, Ultra-low power utilization short reaction time.

\section{CONCLUSION}

All recreations exist made used for binary phase shift keying group of stars, Rayleigh blurring, also using transfers which are permitted to submit blunder in unraveling the transferred information. Maximum- minimum model-based way determination functions admirably for bounces; it keeps away from misfortune in assorted variety because of the nearness of numerous transfers in an agreeable way and empowers the DF convention to accomplish better-assorted variety request by including progressively agreeable ways in the framework.

\section{REFERENCES}

1. J. Boyer, D. D. Falconer, and H. Yanikomeroglu, "Multihop diversity in wireless relaying

2. channels," IEEE Trans. Commun., vol. 52, pp. 18201830 , Oct. 2004.

3. J. N. Laneman, D. N. C. Tse, and G. W. Wornell, "Cooperative diversity in wireless

4. networks: Efficient protocols and outage behavior," IEEE Trans. Inf. Theory, vol. 50, pp. 3062-

5. 3080, Dec. 2004.

6. 3GPP, "Relay architectures for E-UTRA (LTEAdvanced)," TR 36.806, Mar. 2010.

7. S. W. Peters and R. W. Heath, Jr., "The future of WiMAX: Multihop relaying with IEEE

8. 802.16j," IEEE Commun. Magazine, vol. 47, pp. 104111, Jan. 2009.

9. B. Wang, J. Zhang, and A. Høst-Madsen, "On the capacity of MIMO relay channels," IEEE

10. Trans. Inf. Theory, vol. 51, pp. 29-43, Jan. 2005.

11. S. Jin, M. R. McKay, C. Zhong, and K. K. Wong, "Ergodic capacity analysis of amplifying-

12. and-forward MIMO dual-hop systems," IEEE Trans. Inf. Theory, vol. 56, pp. 2204-2224, May.

13. 2010 .

14. X. Tang and Y. Hua, "Optimal design of nonregenerative MIMO wireless relays," IEEE

15. Trans. Wireless Commun., vol. 6, pp. 1398-1407, Apr 2007.

16. O. Mũnoz-Medina, J. Vidal, and A. Agust'in, "Linear transceiver design in nonregenerative relays with channel state information," IEEE Trans. Signal Process., vol. 55, pp.

17. 2593-2604, June 2007

18. W. Guan and H. Luo, "Joint MMSE transceiver design in non regenerative MIMO relay

19. systems," IEEE Commun. Lett., vol. 12, pp. 517-519, July 2008. 